\title{
A stereo disadvantage for recognizing rotated familiar objects
}

\author{
Achille Pasqualotto and William G. Hayward \\ University of Hong Kong, Hong Kong, China
}

\begin{abstract}
We tested recognition of familiar objects in two different conditions: mono, where stimuli were displayed as flat, 2-D images, and stereo, where objects were displayed with stereoscopic depth information. In three experiments, participants performed a sequential matching task, where an object was rotated by up to $180^{\circ}$ between presentations. When the $180^{\circ}$ rotation resulted in large changes in depth for object components, recognition performance in the mono condition showed better performance at $180^{\circ}$ rotations than at smaller rotations, but stereo presentations showed a monotonic increase in response time with rotation. However, $180^{\circ}$ rotations that did not result in much depth variation showed similar patterns of results for mono and stereo conditions. These results suggest that in some circumstances, the lack of explicit 3-D information in 2-D images may influence the recognition of familiar objects when they are depicted on flat computer monitors.
\end{abstract}

Our visual system has developed to recognize objects in a three-dimensional (3-D) world, yet most perceptual studies display static objects on flat computer monitors. To what extent is recognition of objects affected by the presence of explicit 3-D information, such as that gained from stereoscopic disparities? Researchers who have investigated this issue, by displaying stimuli with (stereo) and without (mono) stereoscopic disparities, have generally shown an advantage in recognizing 3-D stimuli over 2-D images (e.g., Edelman \& Bülthoff, 1992). In addition, stereo presentations of objects often assist in generalization to unstudied views. Humphrey and Khan (1992) reported a stereo benefit for recognition of unfamiliar objects that was larger for novel views than for studied views, although this may have been caused by a speed-accuracy trade-off. Farah, Rochlin, and Klein (1994) reported better viewpoint generalization for novel shapes when these were viewed directly (with stereoscopic disparities) than when they were presented via videotape. Burke (2005) and Bennett and Vuong (2006) have both recently reported that generalization to new views of novel objects is better when the objects are depicted in stereo than when depicted in mono, with no evidence of any speed-accuracy trade-off. In addition, Burke, Taubert, and Higman (2007) have shown a similar stereo advantage for generalizing unfamiliar faces to new viewpoints.

Should these results be taken as evidence for a general stereo advantage in object recognition? One concern with such a conclusion is that most of these studies used highly similar objects, either wireclips or deformed torii (Bennett \& Vuong, 2006; Burke, 2005; Edelman \& Bülthoff, 1992) or faces (Burke et al., 2007). This is quite unlike normal recognition of 3-D objects, in which classifications (at least at the basic level of categorization) require discrim- ination among objects quite different in shape, and for which the task is relatively easy, even with 2-D depictions of stimuli. Humphrey and Khan (1992) used qualitatively different novel objects, but, as noted, the evidence for a stereo advantage in their study is weaker.

We wanted to investigate whether there was any benefit of stereo depth when 2-D cues provide good information to support recognition. Recognition of rotated objects is often predicted by changes in the 2-D outline (or silhouette) of an object between study and test views (Hayward, 1998; Hayward, Tarr, \& Corderoy, 1999; Lloyd-Jones \& Luckhurst, 2002). For example, Hayward tested recognition of familiar objects across rotations of $0^{\circ}, 60^{\circ}$, and $180^{\circ}$. Although $180^{\circ}$ was the largest rotation, and so might be expected to produce the largest recognition cost, rotations of this size present similar (if mirror-reversed) outlines of an object when depicted in 2-D; accordingly, performance in this condition was superior to that for the $60^{\circ}$ rotations. Depiction in 3-D introduces a new variation in outline shape when objects are rotated by $180^{\circ}$; a shape component close to the viewer at $0^{\circ}$ will be far from the viewer at $180^{\circ}$. So, although the same component may be visible in both views, the difference in depth will be explicitly coded in 3-D; one might, therefore, expect reduced similarity in recognition performance across a $180^{\circ}$ rotation. ${ }^{1}$ As such, stereo depth information might actually impair object recognition in this situation.

We tested this hypothesis in three experiments that were similar except for the specific views of the objects presented. Each used the same basic methodology as that in Hayward (1998); using familiar objects as stimuli, participants judged whether two sequentially presented stimuli showed the same object or two different objects.

W. G. Hayward, whayward@hku.hk 
If repeated, stimuli might be shown from the same view, or they might be rotated by up to $180^{\circ}$. In Experiments 1 and 2, views were chosen in such a way that across $180^{\circ}$ rotations, there was a reversal of the front-back spatial reference frame (see Figure 1). Our hypothesis was that, because of this reversal, recognition across $180^{\circ}$ rotations would no longer show an advantage over smaller rotations in the stereo condition. Experiment 3 was a control experiment; the initial view showed a side-on view of the object in which there was relatively little variation of the object in depth, and so a $180^{\circ}$ rotation resulted more predominantly in a reversal of the left-right reference frame than in a reversal of front-back relations. Here, we expected the stereo pattern of results to follow the mono pattern.

\section{METHOD}

\section{Participants}

Eighty-four undergraduate students of the University of Hong Kong participated in these experiments in return for payment (36 participated in Experiment 1, 24 in Experiment 2, and 24 in Experiment 3). All reported normal or corrected-to-normal vision. Participants were tested to ensure that they were able to perceive stereo depth by being asked to report the subjective appearance of objects presented with and without stereoscopic depth cues.

\section{Apparatus}

The stimuli used in this experiment consisted of familiar objects (see Figure 1), which were rendered using a professional graphics program (3-D Studio Max). All images showed a grayscale object against a black background and were rendered within a $640 \times$ 480 pixel frame. At each view, the camera was elevated slightly to avoid accidental images, and mono and stereo images were rendered. A mono image was simply a normal rendering of the object from the observed viewpoint. The stereo images were created by placing two cameras with a small displacement either side of the observed viewpoint; these cameras rendered the object such that they contained the disparities necessary for forming a 3-D percept. Although the stereo images were slightly different from the mono image, the perceived viewpoints from which the objects were observed in the mono and stereo conditions were the same. The images were shown to the participants using Stereographics CrystalEyes wireless shutter glasses that were connected to a CRT and a PowerMac G5 computer. In the mono condition, identical images were presented to each eye. The CRT had a refresh rate of $120 \mathrm{~Hz}$ (each eye at $60 \mathrm{~Hz}$ ).

\section{Stimuli}

The labeling of object viewpoints was done on the basis of a $0^{\circ}$ view, which showed the front of an object, and in which the front-back axis ran directly away from the viewer; positive views (e.g., $+30^{\circ}$ ) were counterclockwise rotations in depth from this standard, whereas negative views (e.g., $-30^{\circ}$ ) were clockwise rotations in depth. In Experiment 1, three views were used: a baseline view at $+30^{\circ}$ (roughly canonical; Palmer, Rosch, \& Chase, 1981), and then additional views at $+90^{\circ}$ and $-150^{\circ}$. These views matched those used by Hayward (1998). In Experiment 2, four views were used: $+45^{\circ}$ (baseline), $+105^{\circ},+165^{\circ}$, and $-135^{\circ}$. Experiment 3 also used four views: $-90^{\circ}$ (baseline), $-30^{\circ},+30^{\circ}$, and $+90^{\circ}$. For ease of comparison across experiments, we will generally report the size of the rotation between the first and second presentations of an object, rather than the absolute view of the test presentation.

\section{Design}

Experiment 1 used a $2 \times 2 \times 3$ mixed design with viewing condition order (mono, then stereo, or stereo, then mono) as a betweensubjects factor and viewing condition (mono or stereo) and rotation $\left(0^{\circ}, 60^{\circ}\right.$, or $180^{\circ}$ between the first and second stimuli) as withinsubjects factors. Stimuli were divided into two distinct sets (Set A and Set B) of 20 objects. Each participant performed two blocks of 120 trials, where at the end of the first block, the visual condition was changed (from mono to stereo, or vice versa) and also the objects displayed were changed (from Set A to Set B, or vice versa, ensuring that the specific objects were not repeated across blocks). This design meant that each baseline $\left(+30^{\circ}\right)$ view was encountered eight times across the experiment, and each other view was encountered twice. The order of the blocks was counterbalanced across participants.

In Experiments 2 and 3, viewing condition was a between-subjects factor, and rotation had four levels $\left(0^{\circ}, 60^{\circ}, 120^{\circ}, 180^{\circ}\right)$. In each experiment, 20 objects were used. Each trial was repeated once, resulting in a single block of 320 trials. This resulted in each baseline view being seen 20 times, and in other views being seen 4 times each.

\section{Procedure}

Participants sat in a quiet testing room in front of the computer screen (viewed from a distance of approximately $50 \mathrm{~cm}$ ) and wore shutter glasses. There were four practice trials that familiarized the participants with the task of stating whether two sequential images displayed the same or a different object. The first image was shown after the fixation point marker (a small black cross positioned in the center of the screen for $500 \mathrm{msec}$ ); the first image remained visible for $200 \mathrm{msec}$ and was followed by a mask (consisting of a randomly arranged set of features from across the objects) for $750 \mathrm{msec}$. Then the second image appeared for $100 \mathrm{msec}$ (followed by another mask), and the participants were required to decide (as quickly and as accurately as possible) on the identity of the two stimuli by pressing the corresponding key: "V" for "same" and "M" for "different." Finally, the first block of the main experiment began. In each trial the first image was always from the baseline orientation, and the second image was equally likely to show the same or a different object from one of the possible viewpoints. Participants were asked to respond as quickly as possible while maintaining accuracy. Accuracy and response times (RTs) were recorded. Each experiment took about $30 \mathrm{~min}$ to complete.

\section{RESULTS}

\section{Experiment 1}

RTs for correct responses were averaged for each participant and are summarized in Figure 2. Whereas the pattern for the mono viewing condition replicates the finding of Hayward (1998) in showing maximal viewpoint costs at $60^{\circ}$ and reduced costs at $180^{\circ}$, the stereo viewing condition shows a linear cost function with no major deviation around $60^{\circ}$. These results were analyzed with the use of a three-way mixed ANOVA, with viewing condition order (mono, then stereo; stereo, then mono) as a betweensubjects variable, and viewing condition (mono, stereo) and rotation $\left(0^{\circ}, 60^{\circ}, 180^{\circ}\right)$ as within-subjects variables. The analysis showed a main effect of rotation $[F(2,68)=$ $19.9, p<.001, \eta^{2}=.37$ ] but no main effect of viewing condition $[F(1,34)=2.94, p>.05]$ or order $(F<1$.) Two interactions were statistically significant. The most important of these theoretically was the interaction of viewing condition and viewpoint $[F(2,68)=7.4, p=.001$, $\left.\eta^{2}=.18\right]$, showing a difference in the patterns of viewpoint costs for the mono and stereo conditions. Post hoc Scheffé tests showed that, whereas the mono $180^{\circ}$ rotation was identified significantly more quickly than the mono $60^{\circ}$ rotation, the stereo $180^{\circ}$ rotation was recognized 
A

View 1

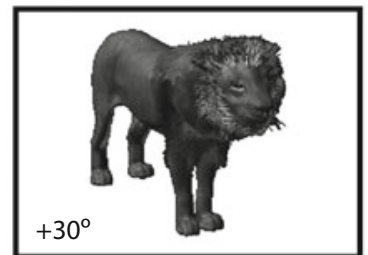

View 2

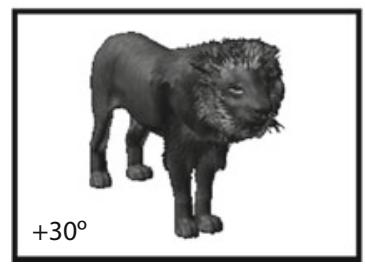

Rotation

B

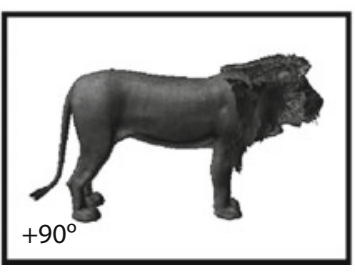

$60^{\circ}$

View 1

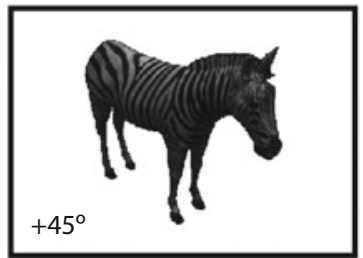

View 2

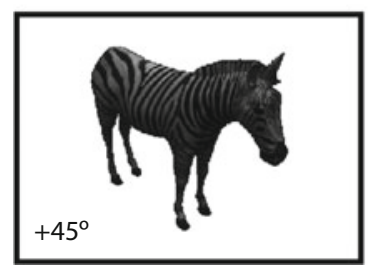

Rotation

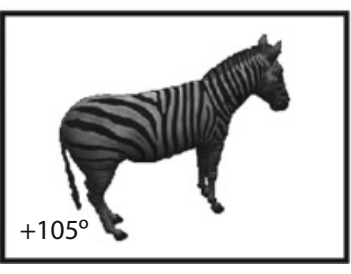

$60^{\circ}$

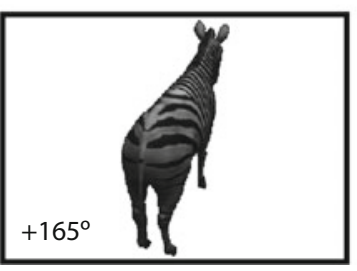

$120^{\circ}$

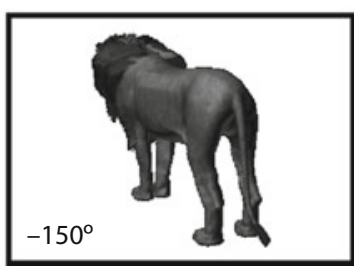

$180^{\circ}$

C

View 1

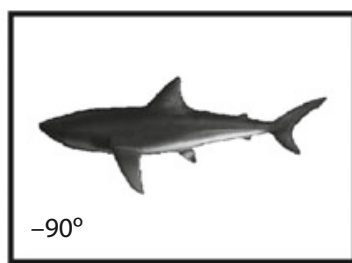

View 2

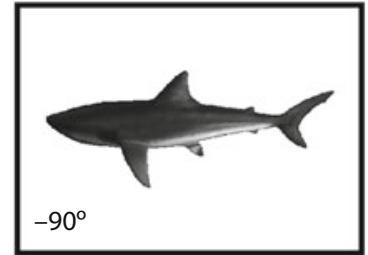

Rotation

$0^{\circ}$

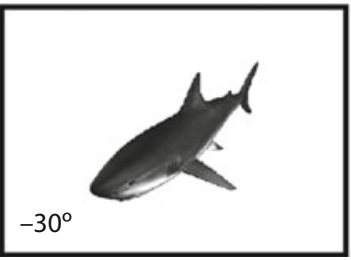

$60^{\circ}$

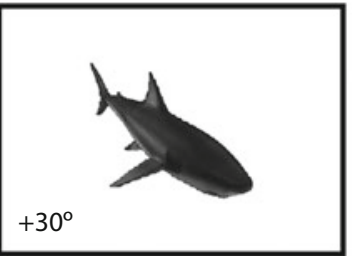

$120^{\circ}$

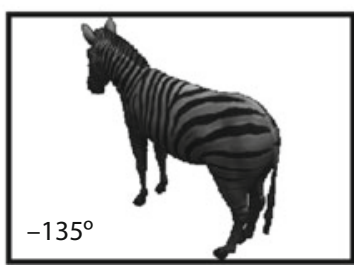

$180^{\circ}$

Figure 1. Stimulus configurations from (A) Experiment 1, (B) Experiment 2, and (C) Experiment 3. In each experiment, the baseline (first) view was presented for $200 \mathrm{msec}$, followed by a 750-msec masked interstimulus interval (ISI), then the second view for $100 \mathrm{msec}$. Absolute viewpoint of each image is shown in the bottom left-hand corner of each frame. 


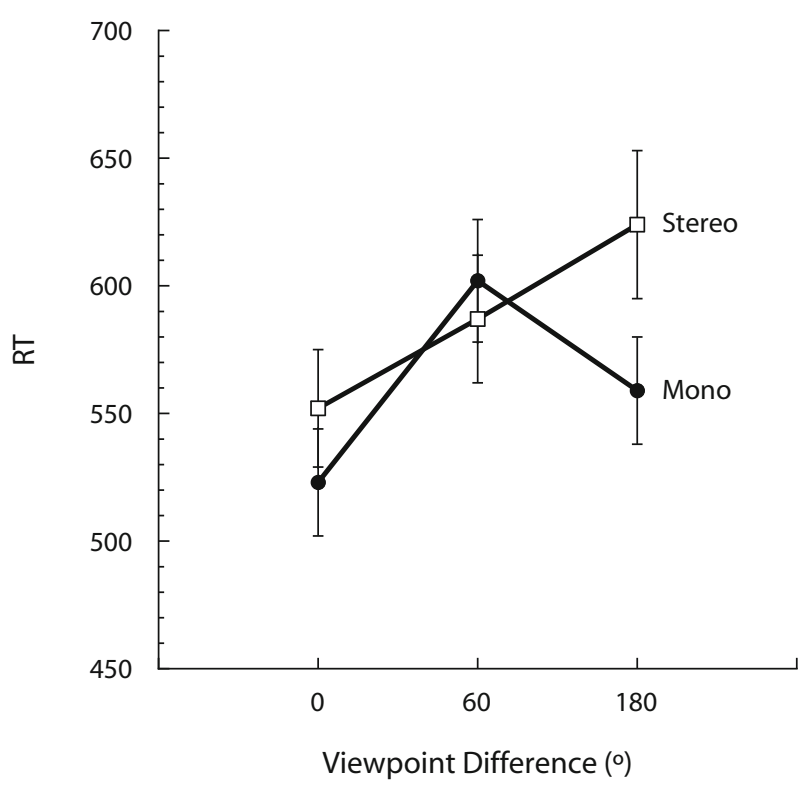

Figure 2. Response times (RTs) from Experiment 1. Error bars show standard errors of the means.

significantly more slowly than the stereo $60^{\circ}$ rotation (all $p \mathrm{~s}<.05$ ). In addition, the mono $180^{\circ}$ rotation was significantly faster than the stereo $180^{\circ}$ rotation $(p<.05)$. The other significant interaction was that of order and viewing condition $\left[F(1,34)=21.09, p<.001, \eta^{2}=.38\right]$, which was caused by faster responses for whichever block was performed second.

This task was very easy, so error rates were low (in all conditions, errors occurred on fewer than $5 \%$ of trials). However, for those errors observed, the pattern of their occurrence (shown in Table 1) was entirely consistent with the analysis of RTs. An ANOVA with the same structure as that performed on the RT data was also conducted for errors. Due to the floor effect, the only statistically significant finding was the main effect for viewpoint $[F(2,68)=$ $\left.9.17, p<.001, \eta^{2}=.21\right]$; the interaction of viewing condition and viewpoint was marginally significant $[F(2,68)=$ $\left.2.45, p<.1, \eta^{2}=.07\right]$.

\section{Experiment 2}

We analyzed the response latencies for correct responses using a two-way mixed ANOVA, with viewing condition (mono or stereo) as a between-subjects variable and rotation $\left(0^{\circ}, 60^{\circ}, 120^{\circ}\right.$, or $\left.180^{\circ}\right)$ as a within-subjects variable. Results were consistent with those in Experiment 1, showing a significant effect of rotation $[F(3,66)=5.34, p<$ $\left..05, \eta^{2}=.2\right]$ and a significant interaction $[F(2,22)=6.79$, $\left.p<.05, \eta^{2}=.24\right]$. There was no main effect of viewing condition $[F(3,66)=1.35, p>.05]$. As in Experiment 1, this analysis shows a different pattern of responses across the viewpoints for the two viewing conditions; mono trials resulted in slower responses across small rotations but an improvement toward $180^{\circ}$, whereas stereo trials showed a monotonically increasing function of response latencies as the size of the rotation increased (see Figure 3). A Scheffé post hoc test confirmed that for mono views, the $60^{\circ}$ rotation produced slower recognition performance than did $0^{\circ}$ $(p<.05)$, whereas for stereo views, the recognition was slower at $180^{\circ}$ than for any other rotation $(p<.05)$. Recognition of the mono $180^{\circ}$ rotation was again faster than that for the stereo $180^{\circ}$ rotation $(p<.05)$.

Error rates were low: the maximal rate was less than $7 \%$ of the trials (see Table 1). The effect of viewpoint was statistically significant $\left[F(3,66)=3.33, p<.05, \eta^{2}=.13\right]$. Due to the floor effect, no other effects reached statistical significance; however, the pattern of responses was consistent with that of latencies.

\section{Experiment 3}

We analyzed response latencies for correct responses using the same ANOVA model as in Experiment 2. As before, we found a significant effect of viewpoint $\left[F(3,66)=11.34, p<.01, \eta^{2}=.34\right]$ and no significant effect of viewing condition $[F(3,66)=2.78, p>.05]$. As opposed to Experiments 1 and 2, however, no significant interaction between viewing condition and viewpoint was found $(F<1)$. In other words, the pattern of costs to response latencies did not vary between mono and stereo conditions (see Figure 4). A Scheffé post hoc test showed that for mono views, the $60^{\circ}$ and $120^{\circ}$ rotations produced slower recognition than did $0^{\circ}$ (both $p \mathrm{~s}<.05$ ); similarly, for stereo views, recognition was slower at $120^{\circ}$ than at $0^{\circ}$ $(p<.05)$.

The maximal error rate was around 4\% (see Table 1). No significant effects were found, since little variation of the data was observed.

\section{DISCUSSION}

In three experiments, we have shown that the presentation of stereo information about an object can affect recognition of that object. In all three experiments, the results of the mono condition replicated the result reported by Hayward (1998): Recognition of familiar objects rotated through $180^{\circ}$ was better than recognition of these objects across shorter $\left(60^{\circ}, 120^{\circ}\right)$ rotations. Indeed, this work extends Hayward's result by demonstrating that such an ad-

Table 1

Mean Errors (As Percentages, With Standard Errors) in the Experiments

\begin{tabular}{|c|c|c|c|c|c|c|c|c|}
\hline & \multicolumn{8}{|c|}{ Rotation } \\
\hline & \multicolumn{2}{|c|}{$0^{\circ}$} & \multicolumn{2}{|c|}{$60^{\circ}$} & \multicolumn{2}{|c|}{$120^{\circ}$} & \multicolumn{2}{|c|}{$180^{\circ}$} \\
\hline & $M$ & $S E$ & $M$ & $S E$ & $M$ & $S E$ & $M$ & $S E$ \\
\hline \multicolumn{9}{|c|}{ Experiment 1} \\
\hline Mono & 1.52 & 0.44 & 4.86 & 0.83 & - & - & 3.60 & 0.84 \\
\hline Stereo & 1.80 & 0.60 & 3.33 & 0.87 & - & - & 5.00 & 1.02 \\
\hline \multicolumn{9}{|c|}{ Experiment 2} \\
\hline Mono & 1.88 & 0.76 & 4.38 & 1.57 & 4.16 & 1.85 & 3.55 & 1.09 \\
\hline Stereo & 2.50 & 1.05 & 3.95 & 1.75 & 4.79 & 1.49 & 6.67 & 1.90 \\
\hline \multicolumn{9}{|c|}{ Experiment 3} \\
\hline Mono & 2.93 & 0.71 & 3.33 & 0.54 & 4.73 & 1.03 & 3.12 & 1.30 \\
\hline Stereo & 2.91 & 1.31 & 3.97 & 1.31 & 4.17 & 1.24 & 4.38 & 1.51 \\
\hline
\end{tabular}




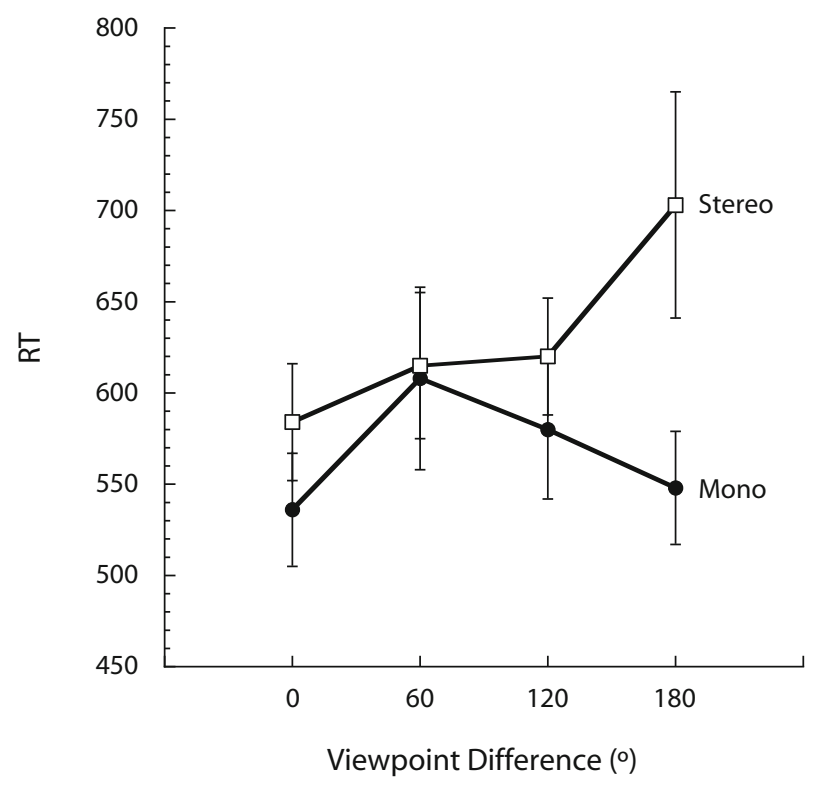

Figure 3. Response times (RTs) from Experiment 2. Error bars show standard errors of the means.

vantage for $180^{\circ}$ rotations is not the result of a particular set of object views (e.g., using $+30^{\circ},+90^{\circ}$, and $-150^{\circ}$ views). However, this pattern was found for stereo stimuli only in Experiment 3. In the other two experiments, recognition of stereo presentations of objects showed a pattern different from that for mono stimuli; rather than performance improving at $180^{\circ}$ rotations, response latencies showed a relatively linear pattern across all viewpoints. On the face of it, this result seems counterintuitive, given that mono stimuli contain a subset of the information present in the stereo condition. Specifically, the key finding in our experiments is that the cue of outline shape seemed to assist performance in Experiments 1 and 2 only in the mono condition; recognition of $180^{\circ}$ rotations in the stereo condition of these experiments seemed to be based on a normalization mechanism more closely aligned with the spatial orientation of the object (e.g., Graf, 2006; Tarr, 1995).

Why do recognition mechanisms switch from using outline shape for mono stimuli (and stereo stimuli in Experiment 3 ) to using a more global normalization mechanism for stereo stimuli in Experiments 1 and 2? The issue is whether 2-D outline shape is easily recoverable. When stimuli are depicted in 2-D in our experiments, the 2-D outline is easily recovered; this leads to better recognition across $180^{\circ}$ rotations than across smaller rotations because the similarity of 2-D outline shape is high in this condition. ${ }^{2}$ However, for stereoscopically defined objects in Experiments 1 and 2, the 2-D outline is harder to recover, because additional variation exists in 3-D; a portion of the outline that was close to the viewer at the baseline view will be far from the viewer at a $180^{\circ}$ rotation. In Experiment 3, the stereoscopic depictions of the object at the baseline view and $180^{\circ}$ rotation have relatively smaller variation in depth because they show side-on views; ${ }^{3}$ here, even in the stereo condition, the 2-D outline is fairly easily recovered, and performance shows the characteristic improvement (relative to intermediate rotations) for both mono and stereo conditions. In order to verify that the 3-D outlines had less variation, and therefore were more similar, across $180^{\circ}$ rotations in Experiment 3 than in Experiments 1 and 2, we tested 20 participants who rated (on a 5 -point scale) the similarity of outlines across 3-D pairs of stimuli used in $180^{\circ}$ rotation trials. Stimuli were rendered as silhouettes with stereoscopic disparities, so that the outline appeared in 3-D. The outlines of $180^{\circ}$ stimulus pairs were perceived as being more similar in Experiment 3 than in the other experiments $[F(3,57)=13.90, p<.001]$; all comparisons were significant with Scheffé post hoc tests, $(p<.05) .{ }^{4}$ Therefore, recognition performance in these experiments, even for very familiar objects, seemed to be sensitive to variation of 3-D shape information.

One concern with the design of the present experiment is its inherent assumption that stereo cues should provide useful shape information to aid in recognition of the objects. A number of studies have shown that stereo information does not give veridical information about object shape; instead, participants systematically misperceive 3-D shape on the basis of stereoscopic disparities (e.g., Johnston, 1991; Lind, Bingham, \& Forsell, 2003; Todd \& Norman, 2003). We do not believe such misperceptions are likely to be the cause of the present results, for a number of reasons. First, such studies typically test shape perception when stereoscopic disparity provides the only shape cue, unlike the present study, in which stereoscopic disparity is added to other, pictorial cues that themselves provide strong shape (and depth) information. Second, misperceptions of 3-D shape from stereo cues are generally quantitative in nature; for example, curvature may be over- or underestimated (Johnston, 1991). Such quantitative misperceptions of shape are

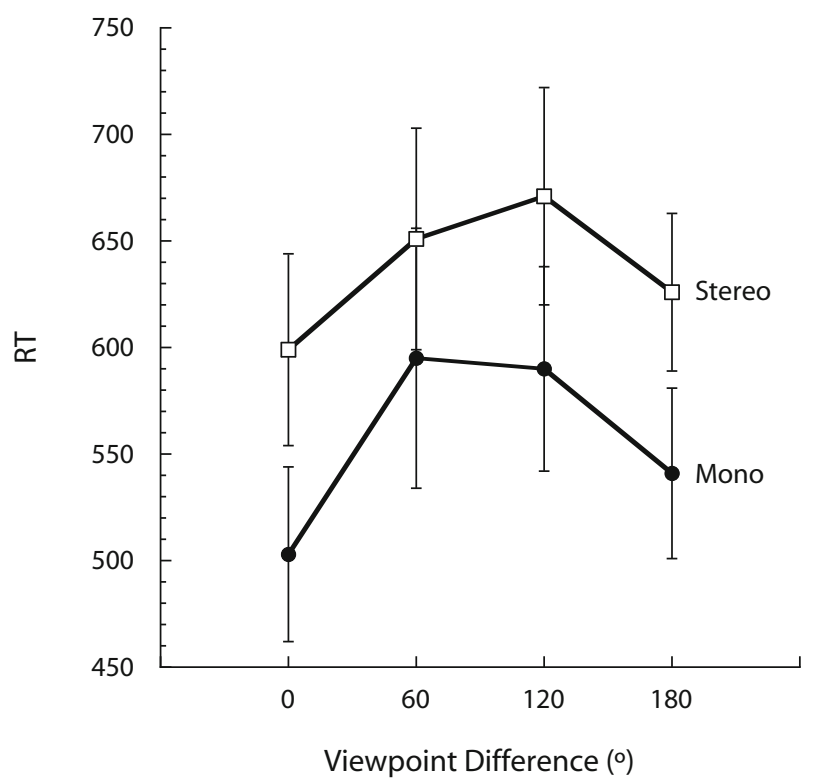

Figure 4. Response times (RTs) from Experiment 3. Error bars show standard errors of the means. 
unlikely to be harmful in the present experiment, since no precise matching of shape is required.

This study seems to suggest that the addition of stereoscopic depth cues impairs judgments of object similarity (when objects have been rotated $180^{\circ}$ ), and as such seems to stand in contrast to research on the integration of pictorial and stereoscopic depth cues to give a unified percept of depth (for a review, see Jacobs, 2002). Whereas differences in the weighting of different cues depend upon the nature of the stimulus configuration (Buckley \& Frisby, 1993), pictorial and stereoscopic depth cues are generally weighted in optimal fashion, on the basis of their reliability (Hillis, Watt, Landy, \& Banks, 2004; Knill \& Saunders, 2003). In these studies, the reliability of different cues is manipulated, and a specific judgment of object shape is required. Our study investigates cue combination somewhat differently; the two conditions differ on the basis of one cue (stereoscopic disparity), and the response on each trial is simply one of object identity, with no precise judgment of shape required. Therefore, the perceptual representation of shape on each trial may well be formed on the basis of an optimal combination of pictorial and stereoscopic depth cues; however, it is in the match of these two representations that the additional stereo cues lead to impaired performance.

To what extent would we expect the results of these three experiments to generalize to other objects? We used familiar objects with axes of bilateral symmetry and showed only one object from each object category. This stands in contrast to many studies of object recognition that employ novel objects and require precise discrimination of shape in order to perform the task; such studies are the basis for most previous investigations of the role of stereoscopic disparity on object recognition (e.g., Bennett \& Vuong, 2006; Burke, 2005) and show a benefit for the provision of stereo depth. Our interpretation is that stereo depth assists in the recovery of precise information about 3-D shape. However, such precise information was unnecessary in our experiments, where more general descriptions of shape were sufficient for performing the task. Here, the extra specificity provided by stereoscopic disparity seems to have obscured more general similarities between the pictorial elements of the images, particularly the outline shapes. We would predict that similar effects will occur for novel objects when more general judgments of shape are required; we are currently investigating this issue.

The results of our experiments can be added to those of earlier studies (e.g., Bennett \& Vuong, 2005; Burke, 2005; Burke et al., 2007) showing that stimuli presented with stereo depth display different patterns of recognition cost over changes in viewpoint from stimuli presented with only pictorial depth cues. However, whereas previous studies reported a benefit in performance with the provision of stereo, we found that it produced a decrement in performance, at least following $180^{\circ}$ rotations. Taken together, these studies point toward the object recognition system's being influenced by 3-D information when objects are encoded; 5 thus, in situations where only 2-D cues are available, such as during psychophysical experiments, observed results may lack some external validity. In our opinion, future experimental methodologies should carefully consider whether any response artifacts may exist that are caused by the removal of explicit depth information (such as stereo and motion). To verify that such artifacts do not exist, it would be useful to test such studies with stereo depictions of objects. If experiments do not control for 2-D cues or use 3-D depictions, their results may not represent valid tests of human object recognition.

\section{AUTHOR NOTE}

This work was supported by a grant from the Hong Kong Research Grants Council (Grant HKU 7649/06H) to W.G.H. Thanks to Sieu Khuu and Joey Cham for assistance in setting up the experiments, and Mike Tarr for providing comments on an earlier version of the manuscript. Correspondence concerning this article should be addressed to W. G. Hayward, Department of Psychology, University of Hong Kong, Pokfulam Road, Hong Kong, China (e-mail: whayward@hku.hk).

\section{REFERENCES}

Bennett, D. J., \& Vuong, Q. C. (2006). A stereo advantage in generalizing over changes in viewpoint on object recognition tasks. Perception \& Psychophysics, 68, 1082-1093.

BuCKLEY, D., \& FRISBY, J. P. (1993). Interaction of stereo, texture, and outline cues in the shape perception of three-dimensional ridges. $\mathrm{Vi}$ sion Research, 33, 919-933. doi:10.1016/0042-6989(93)90075-8

Burke, D. (2005). Combining disparate views of objects: Viewpoint costs are reduced by stereopsis. Visual Cognition, 12, 705-719. doi:10.1080/13506280444000463

Burke, D., TAubert, J., \& Higman, T. (2007). Are face representations viewpoint dependent? A stereo advantage for generalizing across different views of face. Vision Research, 47, 2164-2169. doi:10.1016/j .visres.2007.04.018

Edelman, S., \& Bülthoff, H. H. (1992). Orientation dependence in the recognition of familiar and novel views of three-dimensional objects. Vision Research, 32, 2385-2400. doi:10.1016/0042 -6989(92)90102-O

Farah, M. J., Rochlin, R., \& KleIN, K. L. (1994). Orientation invariance and geometric primitives in shape recognition. Cognitive Science, 18, 325-344.

Graf, M. (2006). Coordinate transformations in object recognition. Psychological Bulletin, 132, 920-945. doi:10.1037/0033 $-2909.132 .6 .920$

HAYWARD, W. G. (1998). Effects of outline shape in object recognition. Journal of Experimental Psychology: Human Perception \& Performance, 24, 427-440. doi:10.1037/0096-1523.24.2.427

Hayward, W. G., Tarr, M. J., \& Corderoy, A. K. (1999). Recognizing silhouettes and shaded images across depth rotation. Perception, 28, 1197-1215. doi:10.1068/p2971

Hillis, J. M., Watt, S. J., LANDY, M. S., \& Banks, M. S. (2004). Slant from texture and disparity cues: Optimal cue combination. Journal of Vision, 4, 967-992. doi:10.1167/4.12.1

Humphrey, G. K., \& Khan, S. C. (1992). Recognizing novel views of three-dimensional objects. Canadian Journal of Psychology, 46, 170190. doi:10.1037/h0084320

JACOBS, R. A. (2002). What determines visual cue reliability? Trends in Cognitive Sciences, 6, 345-350. doi:10.1016/S1364-6613(02)01948-4

Johnston, E. B. (1991). Systematic distortions of shape from stereopsis. Vision Research, 31, 1351-1360. doi:10.1016/0042-6989(91)90056-B

KnILl, D. C., \& SAunders, J. A. (2003). Do humans optimally integrate stereo and texture information for judgments of surface slant? Vision Research, 43, 2539-2558. doi:10.1016/S0042-6989(03)00458-9

Lind, M., Bingham, G. P., \& Forsell, C. (2003). Metric 3D structure in visualizations. Information Visualization, 2, 51-57.

Lloyd-Jones, T. J., \& LUCKHURST, L. (2002). Outline shape is a mediator of object recognition that is particularly important for living things. Memory \& Cognition, 30, 489-498.

Palmer, S., Rosch, E., \& Chase, P. (1981). Canonical perspective and the perception of objects. In J. [B.] Long \& A. [D.] Baddeley (Eds.), Attention and performance IX (pp. 135-151). Hillsdale, NJ: Erlbaum. 
TARR, M. J. (1995). Rotating objects to recognize them: A case study on the role of viewpoint dependency in the recognition of threedimensional objects. Psychonomic Bulletin \& Review, 2, 55-82.

TodD, J. T., \& Norman, J. F. (2003). The visual perception of 3-D shape from multiple cues: Are observers capable of perceiving metric structure? Perception \& Psychophysics, 65, 31-47.

\section{NOTES}

1. In addition, the outline shape of an object may be less salient in an object depicted in 3-D; in 2-D it can be traced as a single contour but in 3-D it becomes a series of contours separated by discontinuities in depth.

2. To check this assumption, similarity of 2-D outlines was computed by comparing silhouettes of the pair of stimuli shown on each trial; in each experiment, the similarity for $180^{\circ}$ rotation pairs was higher than that for pairs of images in the other rotations, as confirmed by Scheffé post hoc tests $(p<.05)$ for each experiment.
3. Assuming that the front-back axis is longer than the left-right axis of an object, when the front-back axis runs parallel to the viewing plane, depth variation will be minimized, since that variation will occur only for left-right shape differences; shape components at the front and back of the object will be at equal distances from the viewer.

4. Four sets of objects were tested: Sets A and B from Experiment 1, along with the sets from Experiments 2 and 3.

5. Although the utility of stereo vision falls off with viewing distance, and is absent for members of the population who are stereo blind, other depth cues that are not present in static 2-D images (such as structurefrom-motion) will still be available. We interpret our result, and others that have been derived from the use of stereoscopic disparity, as being related to the presence of explicit (i.e., nonpictorial) 3-D information in general, rather than being specifically related to mechanisms of stereopsis.

(Manuscript received March 10, 2008; revision accepted for publication May 4, 2009.) 